\title{
Mössbauer transmission and back scattered conversion electron study of Fe nanowires encapsulated in multiwalled carbon nanotubes
}

\author{
T. Ruskov, S. Asenov, I. Spirov, and C. Garcia \\ Institute for Nuclear Research and Nuclear Energy, Tsarigradsko Chaussee 72, BG-1784, Sofia, Bulgaria \\ I. Mönch, ${ }^{\text {a) }}$ A. Graff, ${ }^{\text {b) }}$ R. Kozhuharova, A. Leonhardt, T. Mühl, M. Ritschel, \\ C. M. Schneider, ${ }^{\text {C) }}$ and S. Groudeva-Zotova \\ Leibniz Institute of Solid State and Materials Research Dresden, Helmholtzstrasse, 20, D-01069 Dresden, \\ Germany
}

(Received 15 March 2004; accepted 8 September 2004)

\begin{abstract}
${ }^{57} \mathrm{Fe}$ transmission Mössbauer spectroscopy (TMS) and back scattered conversion electron Mössbauer spectroscopy (CEMS) measurements were carried out on Fe-filled multiwalled carbon nanotubes (Fe-MWCNTs) grown by chemical vapor deposition with ferrocene as precursor. Samples of Fe-MWCNTs material deposited on the inner wall of the quartz tube reactor and samples of aligned Fe-MWCNTs grown perpendicularly to the oxidized Si substrate were characterised by the TMS method. The data show that Fe phases encapsulated within the carbon nanotubes comprise $\alpha$-Fe, $\gamma$-Fe, and $\mathrm{Fe}_{3} \mathrm{C}$ in different percentage ratio depending on the sample preparation. These results are in a good accordance with the previously measured magnetic characteristics and with the structural data found by x-ray diffraction as well by selected area electron diffraction methods and allow a new complementary characterization of the Fe(Fe-alloy)-MWCNT systems. The CEMS method applied for the characterization of metal containing MWCNTs reveals that close to the top surface of the aligned Fe-MWCNTs samples only the $\gamma$-Fe phase is found. This technique shows an additional potential for further investigation of the spatial distribution of the crystalline phases in the depth of aligned Fe-MWCNT samples. (c) 2004 American Institute of Physics.
\end{abstract}

[DOI: $10.1063 / 1.1811781]$

\section{INTRODUCTION}

Recently several papers have addressed the synthesis and properties of Fe-filled carbon nanotubes. ${ }^{1-3}$ These structures may be considered as magnetic nanowires encapsulated in thin carbon shells. Among different applications, they could be used as individual entities in sensors for magnetic scanning probe microscopy and recording heads, as well as for high density magnetic recording media.

Prados et $a l^{2}{ }^{2}$ and Marco et al. ${ }^{3}$ performed Mössbauer spectroscopy and magnetic studies of Fe-filled carbon nanotubes, which were prepared at $1050^{\circ} \mathrm{C}$ by pyrolyzing an 1:1 mixture by weight of ferrocene and $\mathrm{C}_{60}$ under $\mathrm{Ar}$ at atmospheric pressure. The Mössbauer investigation of the obtained carpetlike flakes showed three iron phases being encapsulated within the carbon nanotubes: $\alpha$-Fe, $\gamma$-Fe, and $\mathrm{Fe}_{3} \mathrm{C}$. On the basis of Mössbauer and magnetic measurements the authors proposed a model of spatial distribution of the Fe phases in the nanotubes: a core consisting of elongated $\alpha$-Fe particles, surrounded by the $\gamma$-Fe phase and finally covered by an $\mathrm{Fe}_{3} \mathrm{C}$ layer, which is located at the interface between the metallic phases and the inner wall of the carbon nanotubes.

In our previous work, ${ }^{4,5}$ we reported about the structural

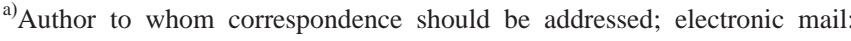
i.moench@ifw-dresden.de

${ }^{b}$ Present address: Max Planck Institute of Microstructure Physics, Halle.

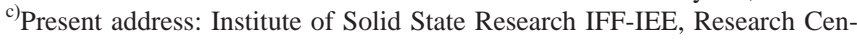
ter Jülich GmbH, Jülich, Germany.
}

and magnetic characteristics of Fe-filled multiwalled carbon nanotubes (MWCNTs) grown by another synthesis routechemical vapor deposition with ferrocene as precursor-on oxidized Si substrates. In this case the Fe-MWCNTs grow aligned perpendicularly to the substrate plane. Varying the deposition conditions Fe-filled nanotubes with relatively uniform core diameters and different thickness of the carbon walls can be obtained. The x-ray diffraction (XRD) and selected area electron diffraction (SAED) analyses of the samples revealed the presence of the same three phases found by the above cited group, ${ }^{2,3}$ however, no satisfying quantitative estimation of the phase percentage was possible by these methods. In addition, no direct experimental results concerning the actual spatial distribution of the Fecontaining phases in the samples have been reported yet, neither in our studies nor in the paper of Prados. ${ }^{2}$ Furthermore, the magnetometry measurements of our samples showed a clear magnetic anisotropy with the easy axis parallel to the axis of the aligned MWCNTs. Most importantly, in contrast to the results of Prados et $a l^{2}{ }^{2}$ and Marco et al. ${ }^{3}$ the low-temperature magnetic behavior of our systems suggested a negligible $\alpha$-Fe/ $\gamma$-Fe exchange coupling. ${ }^{5}$

The aim of this work is to explore the capabilities of the Mössbauer spectroscopy for a quantitative determination of the integral phase composition in the Fe-containing part of the Fe-MWCNTs samples, and to find correlations between these results and the data obtained by other structure sensitive methods. Furthermore, we will also demonstrate the ap- 
TABLE I. Preparation conditions of the studied Fe-MWCNTs samples.

\begin{tabular}{cccc}
\hline \hline Sample & $\begin{array}{c}T_{1} \\
\left({ }^{\circ} \mathrm{C}\right)\end{array}$ & $\begin{array}{c}T_{2} \\
\left({ }^{\circ} \mathrm{C}\right)\end{array}$ & $\begin{array}{c}\mathrm{Ar} \\
(\mathrm{SCCM})^{\mathrm{a}}\end{array}$ \\
\hline $\begin{array}{c}\text { Nanotubes grown on the } \\
\text { walls of the reactor }\end{array}$ & 120 & $850-900$ & 100 \\
\hline $\begin{array}{c}\text { Nanotubes grown } \\
\text { perpendicularly to the } \\
\text { silicon substrate } 4 \times 4 \mathrm{~mm}^{2} \\
\quad \text { (SA-I) }\end{array}$ & 200 & 950 & 200 \\
\hline $\begin{array}{c}\text { Nanotubes grown } \\
\text { perpendicularly to the } \\
\text { (SA-II) }\end{array}$ & & & \\
silicon substrate $10 \times 10 \mathrm{~mm}^{2}$ & 140 & 880 & \\
\hline
\end{tabular}

${ }^{\mathrm{a}}$ Cubic centimeter per minute at standard temp. and pressure.

plication of the conversion electron Mössbauer spectroscopy (CEMS) method for studying Fe-containing phases on the cap layer of aligned samples.

In the following parts of this paper we will present and discuss results from ${ }^{57} \mathrm{Fe}$ Mössbauer spectroscopy measurements of two types of Fe-containing MWCNT samples: (i) carpetlike material, deposited on the inner wall of the quartz reactor tube and (ii) two samples of Fe-MWCNTs aligned perpendicularly to the oxidized Si substrate (SA-I and SA-II) which were grown under different process conditions. The CEMS analysis has been performed on the latter samples.

\section{EXPERIMENT}

\section{A. Preparation and preliminary characterization}

The samples with Fe-filled carbon nanotubes were prepared by the pyrolysis of ferrocene. ${ }^{4}$ The nanotubes were grown in a quartz tube reactor inside a dual zone furnace system. The main parameters of the deposition process are the temperature of the first furnace zone for the sublimation of the ferrocene $\left(T_{1}\right)$, the gas flow rate and the growth temperature in the second furnace zone $\left(T_{2}\right)$. The process was performed as follows. In the first zone the ferrocene was sublimated at temperatures of about $120-200{ }^{\circ} \mathrm{C}$ and transported into the second furnace (the hot reaction zone) by a controlled Ar flow. At high temperature $\left(T_{2}=880-950{ }^{\circ} \mathrm{C}\right)$ the nanotubes were formed on the walls of the quartz tube reactor, or on silicon substrates placed in the reaction zone. The selected three samples of Fe-filled MWCNTs are obtained at different conditions which are shown in Table I.

In Fig. 1 scanning electron microscopy (SEM) images of the obtained material are presented. The nanotubes grown on the walls of the reactor consist mainly of a mixture of bundles of well-aligned Fe-filled MWCNT and metal particles encapsulated by carbon [Fig. 1(a)], whereas the CNT grown onto the silicon substrates exhibit a distinct alignment perpendicular to the substrate surface [Fig. 1(b)]. In both cases the nanotubes have outer diameters of 40-100 nm and lengths up to $30 \mu \mathrm{m}$. The diameters of the metal cores are in the range of 20-40 nm. The walls consist of cylindrical graphite (002)-lattice planes parallel to the tube axis with a lattice plane distance of about $0.34 \mathrm{~nm}^{4}$

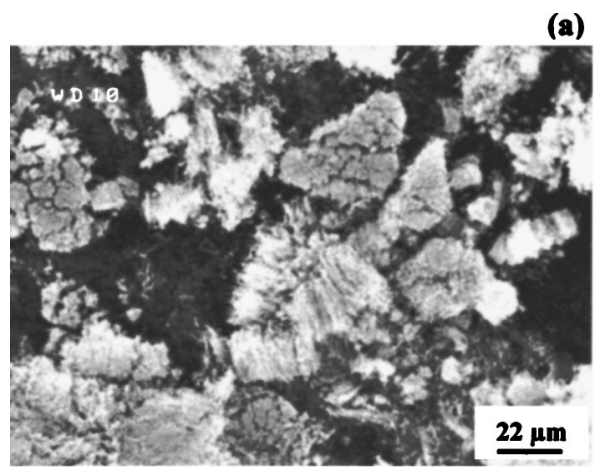

(b)

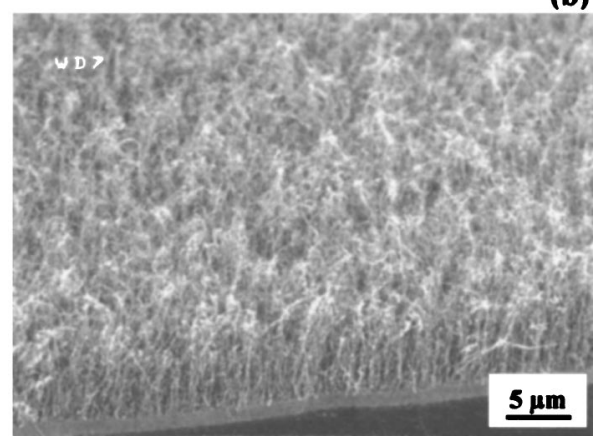

FIG. 1. SEM images of well-aligned Fe-filled MWCNT grown on the walls of the reactor (a) and on an oxidized silicon substrate perpendicular to the substrate surface (b).

On several individual nanotubes we carried out a local structural characterization by means of SAED. The studies revealed that the nanotubes contain single crystals of the ferromagnetic bcc $(\alpha-\mathrm{Fe})$ and the paramagnetic fcc $(\gamma-\mathrm{Fe})$ structure (Fig. 2). The SAED patterns of two nanowires encapsulated inside the carbon nanotubes are shown as an inset in Fig. 2. The spots in the electron diffraction pattern in Fig. 2(a) correspond to the bcc Fe phase, whereas these in Fig. 2(b) originate from the fcc Fe lattice.

The integral phase composition of the samples has been determined by XRD analysis in Bragg-Brentano geometry using a Co $K_{\alpha}$ radiation. The XRD patterns of the three samples chosen for the Mössbauer spectroscopy investiga- (a)

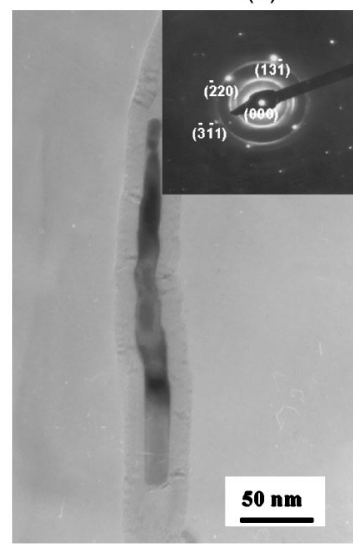

(b)

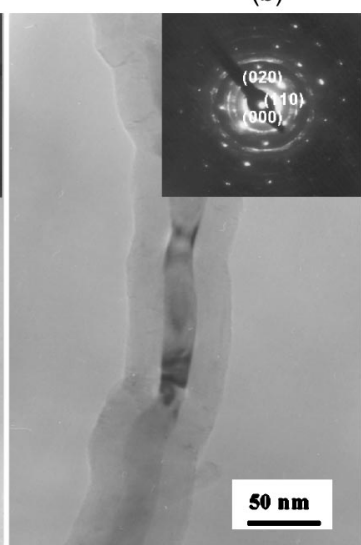

FIG. 2. TEM images of Fe nanowires encapsulated inside the nanotubes. The insets are the measured SAED patterns of the corresponding nanowires showing the presence of bcc Fe structure (a) and fcc Fe structure (b). 


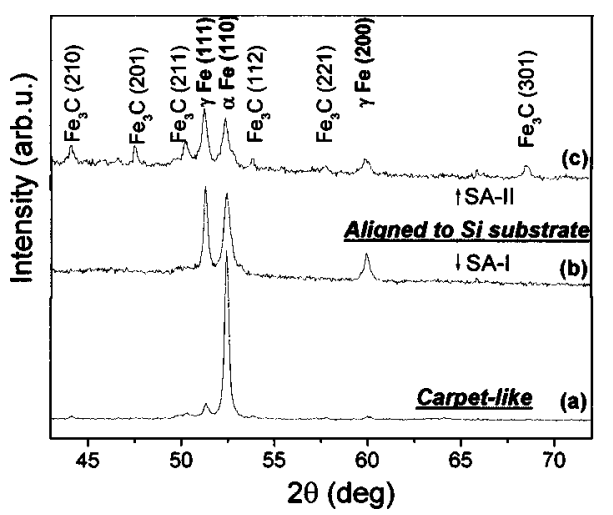

FIG. 3. XRD patterns of the investigated Fe-filled MWCNT: (a) Carpetlike sample taken from the reactor walls - containing a dominating high textured $\alpha$-Fe phase; (b) aligned sample SA-I containing a mixture of the high (110) textured $\alpha$-Fe and the weakly-(111) textured $\gamma$-Fe; (c) aligned sample SA-II containing a mixture of $\alpha$ - $\mathrm{Fe}, \gamma$-Fe, and $\mathrm{Fe}_{3} \mathrm{C}$ phases.

tions show the presence of three different Fe-containing crystalline phases with varying abundance-the $\alpha$-Fe phase with a very high (110) texture, the $\gamma$-Fe phase with a weaker (111) texture, and a not textured carbide phase (see Fig. 3). According to these results, the relative quantity of the individual phases in the investigated samples is quite different: a dominating $\alpha$-Fe phase in the carpetlike sample grown on the reactor walls-Fig. 3(a); coexsisting $\alpha$-Fe and $\gamma$-Fe phases in the sample grown on Si substrate (SA-I) - Fig. 3(b) and a phase mixture of $\alpha$-Fe, $\gamma$-Fe, and $\mathrm{Fe}_{3} \mathrm{C}$ in the second sample grown on Si substrate (SA-II) - Fig. 3(c). The found $\mathrm{Fe}_{3} \mathrm{C}$ phase is reported for another similar samples and discussed by different authors ${ }^{6-8}$ as a necessary intermediate product appearing during the MWCNTs growth. However, the XRD spectra do not allow a satisfying quantitative characterization of the phase composition in these samples.

\section{B. Mössbauer spectroscopy}

The sample of the Fe-MWCNT material formed on the inner wall of the quartz tube was prepared as follows. A hole with a diameter of $4 \mathrm{~mm}$ made into a Plexiglas disk was filled with the material of Fe-MWCNTs. A small drop of $1.3 \%$ polyvinyl-alcohol water solution was put in the hole. After mixing and drying a bonded powder sample of specific weight $20 \mathrm{mg} / \mathrm{cm}^{2}$ was obtained.

Two samples of aligned Fe-filled multiwalled carbon nanotubes grown on substrates with dimensions $4 \times 4 \mathrm{~mm}^{2}$ (SA-I) and $10 \times 10 \mathrm{~mm}^{2}$ (SA-II) were studied as well. The $\mathrm{Si}$ substrate (thickness $275 \mu \mathrm{m}$ ) is sufficiently transparent for the $14.4 \mathrm{keV} \gamma$ line. Mössbauer spectra were taken in the transmission, as well as in the backscattered conversion electron mode with a conventional constant acceleration spectrometer. $\mathrm{A}{ }^{57} \mathrm{Co}(\mathrm{Rh})$ source with a diameter of the activity spot of $4 \mathrm{~mm}$ was used. The conversion electron Mössbauer spectroscopy measurements were carried out with a $\mathrm{He}$ gas flow proportional counter. ${ }^{9}$ All spectra were fitted using an integral Lorentzian line shape approximation. ${ }^{10,11}$ This approach permits us to separate the absorber (scatterer) line widths and to avoid the saturation thickness effect. The parameters of the fitted spectra corresponding to isomer shift (IS), quadrupole shift (QS), magnetic field at the site of the Fe nucleus $(H)$, and relative spectral area are shown in Table II. The isomer shifts of the spectra are referred to the centroid of an $\alpha$-Fe foil reference spectrum at room temperature (RT). The geometric effect due to the source motion is taken into account as well.

\section{RESULTS AND DISCUSSION}

\section{A. "Powder" Fe-MWCNT sample (carpetlike material) grown on the reactor wall}

Two transmission Mössbauer spectra for the FeMWCNT "powder" sample were taken at both room temperature and at $77 \mathrm{~K}$. They are shown in Fig. 4. In the figures the spectral signatures from three iron phases are seen: two sextets (sx1 and sx2) corresponding to $\alpha$-Fe and $\mathrm{Fe}_{3} \mathrm{C}$, respectively, and a singlet (sn), corresponding to $\gamma$-Fe. The first two phases relate to a ferromagnetic state, while the

TABLE II. Mössbauer parameters obtained from the fit of the spectra.

\begin{tabular}{|c|c|c|c|c|c|}
\hline Sample & Component & $\begin{array}{c}\mathrm{IS} \\
(\mathrm{mm} / \mathrm{s})\end{array}$ & $\begin{array}{c}\mathrm{QS} \\
(\mathrm{mm} / \mathrm{s})\end{array}$ & $\begin{array}{c}\mathrm{H} \\
(\mathrm{kOe})\end{array}$ & $\begin{array}{c}\text { Relative area } \\
\qquad(\%)\end{array}$ \\
\hline \multirow{3}{*}{ Powder (RT) } & sx1 & 0 & 0 & $331.6 \pm 0.1$ & $83.6 \pm 0.4$ \\
\hline & $\mathrm{sx} 2$ & $0.21 \pm 0.01$ & $0.05 \pm 0.01$ & $207.8 \pm 0.6$ & $9.34 \pm 0.4$ \\
\hline & sn & $-0.12 \pm 0.01$ & $\ldots$ & $\ldots$ & $7.07 \pm 0.2$ \\
\hline \multirow{3}{*}{ Powder (77 K) } & sx1 & $0.12 \pm 0.01$ & 0 & $336.1 \pm 0.1$ & $83.7 \pm 0.4$ \\
\hline & $\mathrm{sx} 2$ & $0.31 \pm 0.01$ & 0 & $246.4 \pm 0.5$ & $9.9 \pm 0.3$ \\
\hline & sn & $0.02 \pm 0.01$ & $\cdots$ & $\ldots$ & $6.4 \pm 0.2$ \\
\hline Aligned (RT) & sx1 & 0 & 0 & $330.4 \pm 0.6$ & $58 \pm 3$ \\
\hline SA-I & sn & $-0.10 \pm 0.01$ & $\cdots$ & $\ldots$ & $42 \pm 1$ \\
\hline & sx1 & 0 & 0 & $330.4 \pm 0.6$ & $14 \pm 2$ \\
\hline Aligned (RT) & $\mathrm{sx} 2$ & $0.18 \pm 0.01$ & 0 & $205.8 \pm 0.4$ & $62 \pm 2$ \\
\hline SA-II & sn & $-0.12 \pm 0.01$ & $\cdots$ & $\ldots$ & $24 \pm 1$ \\
\hline $\begin{array}{c}\text { Aligned (RT) } \\
\text { (CEMS) } \\
\text { SA-II }\end{array}$ & sn & $-0.09 \pm 0.01$ & $\cdots$ & $\cdots$ & 100 \\
\hline
\end{tabular}




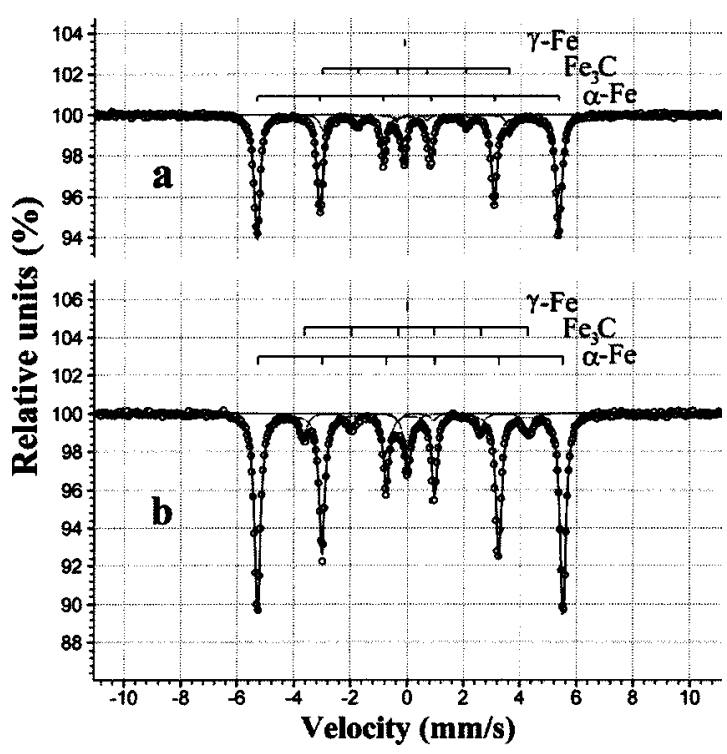

FIG. 4. Mössbauer spectrum of Fe-filled MWCNT powder sample at room temperature (a) and at $77 \mathrm{~K}(\mathrm{~b})$

third corresponds to a paramagnetic state. The Néel temperature of $\gamma$-Fe is estimated to about $60 \mathrm{~K} .^{12}$ The relative spectral areas corresponding to these three phases at room temperature are $\alpha$-Fe (84\%), $\mathrm{Fe}_{3} \mathrm{C}(9 \%)$, and $\gamma$-Fe (7\%). Nearly the same ratio of the spectral areas for the iron components is obtained from the spectrum at $77 \mathrm{~K}$ : $\alpha$-Fe $(84 \%), \mathrm{Fe}_{3} \mathrm{C}$ $(10 \%)$, and $\gamma-\mathrm{Fe}(6 \%)$. These results show that with a sufficient accuracy the Mössbauer-Lamb factors for the corresponding phases increase by the same factor when lowering the temperature. This allows us to consider the area percentage ratio, derived from the fitted experimental spectra (integral approximation) as percentage ratio for the iron phases. Slight broadening (30\%) is observed for the $\gamma$-Fe line at $77 \mathrm{~K}$ with respect to the line width at room temperature, presumably because we come close to the Neel temperature of $\gamma$-Fe. The preservation of the relative spectral areas for the individual iron components with decreasing temperature indicates the absence of sizeable superparamagnetic $\alpha$-Fe and $\mathrm{Fe}_{3} \mathrm{C}$ fractions that might otherwise contribute to the singlet spectrum of $\gamma-\mathrm{Fe}$ at room temperature. Since the carbon nanotubes in the sample are oriented randomly, one should expect the ratio for the relative intensities of the six absorption lines of the sextets as $3: 2: 1: 1: 2: 3,{ }^{13,14}$ which indeed has been obtained for this sample.

\section{B. Samples with aligned Fe-MWCNT grown on oxidized Si substrates}

\section{Transmission Mössbauer spectroscopy}

Mössbauer spectroscopy measurements at room temperature in transmission and backscattered conversion electron mode were carried out for two samples of Fe-MWCNTs, aligned perpendicularly to the oxidized Si (100) substrate, which have been prepared at different conditions (see Table I). The propagation direction of the $\gamma$ rays coincides with the axis of the aligned nanotubes. The transmission Mössbauer spectrum of the first sample (SA-I) is shown in Fig. 5. It contains only the $\alpha$-Fe sextet and the $\gamma$-Fe singlet with a

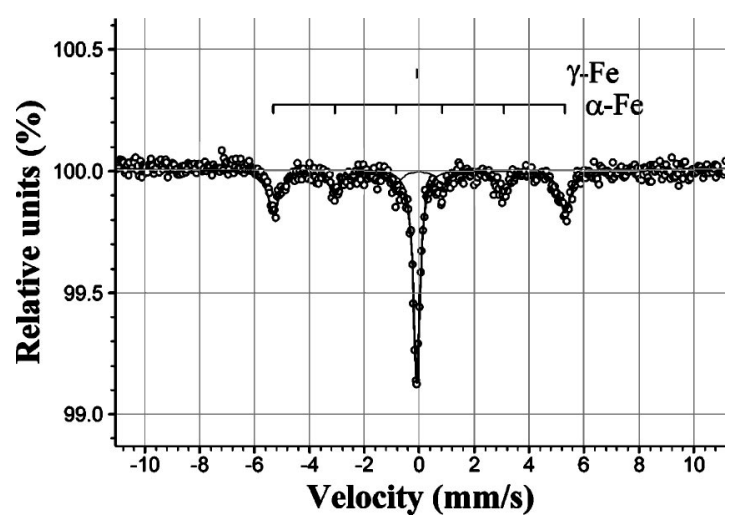

FIG. 5. Mössbauer spectrum of aligned Fe-filled MWCNTs (SA-I).

percentage ratio of the two components of 58\%:42\%, respectively. The relative intensities of the six absorption lines for the ferromagnetic $\alpha$-Fe phase follow a ratio $3: 1.7: 1: 1: 1.7: 3$. That means, there is a slight orientation of the remanent magnetization along the axis of the aligned nanotubes. ${ }^{13,14}$ The transmission Mössbauer spectrum of the second sample (SA-II) is shown in Fig. 6. It contains: $\alpha$-Fe sextet (14\%), $\mathrm{Fe}_{3} \mathrm{C}$ sextet (62\%), and $\gamma$-Fe singlet (24\%). Within the frame of the experimental error we do not see any preferred orientation of the magnetization for this sample, neither for $\alpha$-Fe nor for $\mathrm{Fe}_{3} \mathrm{C}$.

It should be mentioned that the absence of iron carbide in (SA-I) and its presence in (SA-II) correlates with different conditions of their preparation. Relatively lower temperatures of the first furnace zone $\left(T_{1}\right)$ and in the second hot zone $\left(T_{2}\right)$, and also relatively lower Ar flow rate promote the forming of $\mathrm{Fe}_{3} \mathrm{C}$ (SA-II). In contrast higher values of these parameters determine the absence of iron carbide phase (SAI). This statement is in accordance with the absence of $\mathrm{Fe}_{3} \mathrm{C}$ in Fe-MWCNTs powder sample (carpetlike material) as well, derived from Mössbauer spectroscopy measurements (see Tables I and II).

\section{Conversion electron Mössbauer spectroscopy}

In ${ }^{57} \mathrm{Fe}$ conversion electron Mössbauer spectroscopy the detection of backscattered conversion electrons is often used for the study of the surface and layers in the immediate vicinity of the surface. In our experiments we used a gas flow proportional counter, ${ }^{9}$ in which case large counting rates can

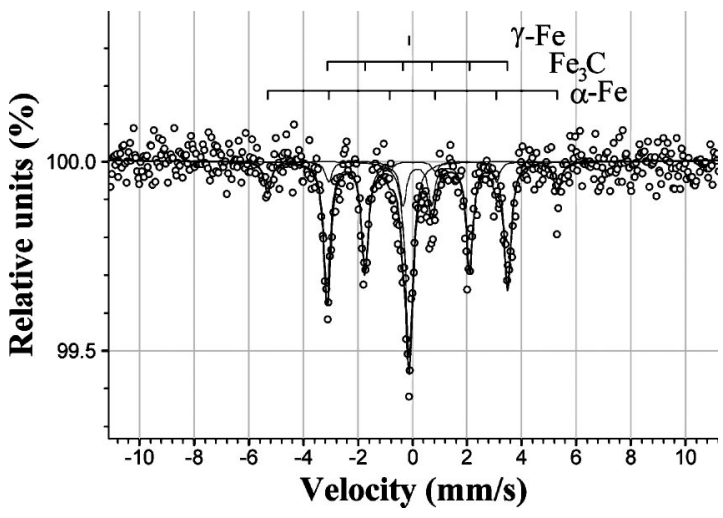

FIG. 6. Mössbauer spectrum of aligned Fe-filled MWCNTs (SA-II). 


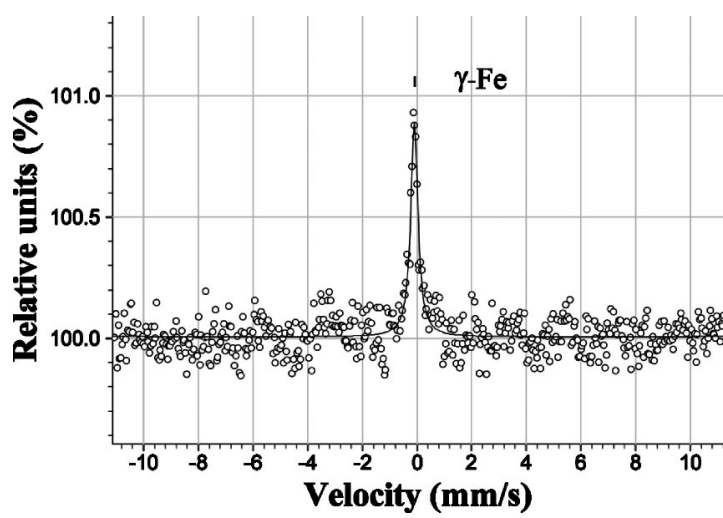

FIG. 7. CEM spectrum of aligned Fe-filled MWCNTs (SA-II).

be obtained. Although the energy resolution of proportional counter is much poorer than that of vacuum electron spectrometers, in some cases it is sufficient for a rough separation of the conversion electrons emitted by the layers in the immediate vicinity of the surface from the electrons emitted by deeper layers. ${ }^{15}$

The conversion electron Mössbauer spectra for both samples of MWCNTs, aligned perpendicular to the Si (100) substrate is characterized by only the $\gamma$-Fe singlet (see Fig. 7). Thus, within the statistical error of our experiment we can conclude there are no ferromagnetic $\alpha$-Fe and iron carbide contributions in the aligned Fe-MWCNTs samples down to a depth less than $200 \mathrm{~nm}$ from the top surface. (The above depth is an estimation based on the effective thickness of escaping of conversion electrons for metallic iron, see, e.g., Ref. 9). We also mention that within the statistical error of our experiment we do not see any admixtures of $\alpha$-Fe and $\mathrm{Fe}_{3} \mathrm{C}$ in superparamagnetic state, which would give rise to a different line shape of the singlet spectrum. Apparently, the top layer of the nanotube films down to a depth of at least $200 \mathrm{~nm}$ contains only the paramagnetic $\gamma$-Fe phase. This peculiarity may be associated with a change in the nanotube growth process, when the ferrocene flow is reduced at the end of the pyrolysis stage.

At present, these data do not allow a quantitative argument on the abundance of $\gamma$-Fe in the top layer of the film and in the bulk, because the area percentage values from transmission Mössbauer spectroscopy (TMS) and CEMS cannot be directly compared. As has been mentioned above, the SAED analysis [Fig. 2(b)] has revealed that some of the crystalline $\mathrm{Fe}$ grains within the tubes also have a fcc structure corresponding to the $\gamma$-Fe phase. Unfortunately, the SAED analysis gives only a very local information with small statistical significance.

In this respect, complementary experiments including a series of ion etching procedures of the MWCNT sample and subsequent conversion electron Mössbauer spectroscopy experiments can be very useful. Such experiments are under way and we expect that they will provide additional information about the spatial distribution of the Fe phases inside the Fe-filled CNT samples and thus, about the properties of this interesting materials class.

\section{CONCLUSIONS}

We have shown the application of ${ }^{57} \mathrm{Fe}$ transmission and back scattered conversion electron Mössbauer spectroscopy methods to Fe-filled multiwalled carbon nanotubes that are grown by chemical vapor deposition with ferrocene as precursor. In particular, we have determined the relative amount of the iron-containing phases in the bulk and on the top layer of the aligned samples. The results confirm and complement the structural data of the samples obtained by XRD and SAED analyses.

The use of the CEMS measurement for investigation of aligned Fe-MWCNTs samples has given detailed information about the upper $200 \mathrm{~nm}$ surface layer where only the $\gamma$-Fe phase has been found and has demonstrated a promising application of this method for the investigation of the spatial distribution of the phases in the Fe filling. Such investigations in the future can provide very important information about the possible interaction between the different $\mathrm{Fe}$ phases $^{2,3}$ and about the origin of the differences in the lowtemperature magnetic behavior of the Fe-MWCNTs found by different groups. ${ }^{2-5}$

\section{ACKNOWLEDGMENTS}

Financial support provided by the Deutsche Forschungsgemeinschaft through Grant No. Fi 439/9 and Grant No. 436BUL 112/39/03 was gratefully acknowledged. The authors would like to thank Nikolay Radoslavov and Teodor Takov for their help during Mössbauer measurements.

${ }^{1}$ N. Grobert et al., Appl. Phys. Lett. 25, 3363 (1999).

${ }^{2}$ C. Prados et al., Phys. Rev. B 65, 113405 (2002).

${ }^{3}$ J. F. Marco et al., Hyperfine Interact. 139/140, 535 (2002).

${ }^{4}$ A. Leonhardt et al., Diamond Relat. Mater. 12, 790 (2003).

${ }^{5}$ T. Mühl et al., J. Appl. Phys. 93, 1 (2003).

${ }^{6}$ N. Grobert et al., Appl. Phys. A: Mater. Sci. Process. 67, 595 (1998).

${ }^{7}$ M. Mayne, N. Grobert, M. Terrones, R. Kamalakaran, M. Rühle, H. W.

Kroto, and D. R. M. Walton, Chem. Phys. Lett. 338, 101 (2001).

${ }^{8}$ S. Groudeva-Zotova et al., J. Magn. Magn. Mater. (to be published).

${ }^{9}$ T. Ruskov, R. Ruskov, D. Paneva, and D. Lefterov, Nucl. Instrum. Methods Phys. Res. B 159, 60 (1999).

${ }^{10}$ G. K. Shenoy, J. M. Friedt, H. Maleta, and S. L. Ruby, Mössbauer Effect Methodology 9, 277 (1974).

${ }^{11}$ T. E. Cranshaw, J. Phys. E 7, 122 (1974).

${ }^{12}$ U. Gonser, C. J. Meechan, A. H. Muir, and H. Wiedersish, J. Appl. Phys. 34, 2373 (1963).

${ }^{13}$ G. K. Werthiem, Mössbauer Effect: Principles and Applications, (Academic, New York, 1964).

${ }^{14}$ K. Haneda, Can. J. Phys. 65, 1233 (1987).

${ }^{15}$ T. Ruskov, A. Rastanawi, and A. Strelkov, J. Phys. D 28, 147 (1995). 